\title{
Platelet-rich plasma promotes the migration and invasion of synovial fibroblasts in patients with rheumatoid arthritis
}

\author{
SHANSHAN YAN ${ }^{1-3^{*}}$, BINZHOU YANG $^{1 *}, \mathrm{CHEN} \mathrm{SHANG}^{4}$, ZHONGSHUANG MA $^{1}$, \\ ZIZHENG TANG ${ }^{1}$, GUIPING LIU ${ }^{1}$, WEIGAN SHEN ${ }^{1,5,6}$ and YU ZHANG ${ }^{1,2,5,6}$ \\ ${ }^{1}$ School of Medicine; ${ }^{2}$ Clinical Medical College, Yangzhou University, Yangzhou, Jiangsu 225001; \\ ${ }^{3}$ Center for Medical Ultrasound, Suzhou Municipal Hospital, Nanjing Medical University Affiliated Suzhou Hospital, \\ Suzhou, Jiangsu 215002; ${ }^{4}$ Department of Rheumatology, The First People's Hospital of Lianyungang, Lianyungang, \\ Jiangsu 222000; ${ }^{5}$ Jiangsu Key Laboratory of Integrated Traditional Chinese and Western Medicine for Prevention \\ and Treatment of Senile Diseases, Yangzhou, Jiangsu 225001; ${ }^{6}$ Jiangsu Co-innovation Center for Prevention and \\ Control of Important Animal Infectious Diseases and Zoonoses, Yangzhou, Jiangsu 225009, P.R. China
}

Received June 25, 2015; Accepted July 6, 2016

DOI: $10.3892 / \mathrm{mmr} .2016 .5500$

\begin{abstract}
Platelet-rich plasma (PRP) is blood plasma that has been enriched with platelets, and the number of platelets is correlated with rheumatoid activity. PRP is a concentrated source of autologous platelets, and contains several different growth factors and cytokines, including platelet-derived growth factor, transforming growth factor- $\beta$ and insulin-like growth factor-1, which stimulate healing of bone and soft tissue. Rheumatoid arthritis (RA) is characterized by synovial hyperplasia, cell activation, articular inflammation and invasion of the synovium into the adjacent bone and cartilage. The adhesion of fibroblast-like synoviocytes (FLSs) onto the extracellular matrix (ECM), migration and invasion are important for the erosion and destruction of the articular cartilage of patients with RA. The aim of the present study was to investigate the effects of PRP on the adhesion, migration and invasion of RA-FLSs. Scratch and Transwell migration assays determined that PRP at a concentration of 2 and $5 \%$ significantly enhanced the migration ability of RA-FLSs. Treatment of RA-FLSs with 2 and 5\% PRP promoted the adhesion and invasion of the cells. Additionally, the immunofluorescence assay revealed that PRP induced a decrease in the number of centrally located stress fibers and led to an increase in the formation of filopodia and lamellipodia in the
\end{abstract}

Correspondence to: Professor Yu Zhang or Professor Weigan Shen, School of Medicine, Yangzhou University, 11 Huaihai Road, Yangzhou, Jiangsu 225001, P.R. China

E-mail: yzzy10182001@aliyun.com

E-mail: shenweigan@hotmail.com

*Contributed equally

Key words: platelet-rich plasma, synovial fibroblasts, rheumatoid arthritis, migration, invasiveness detectable leading edge protrusions in RA-FLSs. In addition, reverse transcription-quantitative polymerase chain reaction and western blot analysis determined that PRP upregulated the protein and mRNA expression levels of matrix metalloproteinase-1 (MMP-1). In conclusion, the promotion of RA-FLS cell migration, invasion and adhesion on the ECM by PRP may be modulated through the upregulation of MMP-1 expression and the induction of actin cytoskeletal reorganization.

\section{Introduction}

Rheumatoid arthritis (RA) is a chronic immune-mediated disease that affects $\sim 1 \%$ of the world's population. It is characterized by synovial hyperplasia, activation of RA-fibroblast-like synoviocytes (FLSs), articular inflammation and invasion of the synovium into the adjacent bone and cartilage (1-3). It has been reported that macrophages, B-cells, T-cells, chondrocytes and osteoclasts are involved in the pathogenesis of RA (4-8). A previous study determined that activated RA-FLSs present in the rheumatoid synovium are crucial for the progression of RA (9). Subsequent to activation, RA-FLSs produce various cytokines, chemokines and matrix-degrading enzymes that mediate the interaction with neighboring inflammatory and endothelial cells and lead to the progressive degradation of the articular cartilage and bone (10). RA starts in a few joints; however, it may spread to all of the joints during the development of the disease, depending on the migration ability of the RA-FLSs. They are able to migrate long distances through the blood stream and move towards, attach to and invade distant exposed cartilage matrix (11). Additionally, RA-FLSs develop a unique aggressive phenotype that leads to increased invasiveness into the extracellular matrix (ECM), exacerbating joint damage $(12,13)$.

Platelet-rich plasma (PRP) is autologous plasma that has a platelet concentration that is elevated three to four times above the baseline levels (13-15). It has been established that the number of platelets is correlated with rheumatoid activity. In addition, a patient with RA may have an increasing 
number of platelets during the active stages of the disease, which may be reduced with the decrease in inflammation (16). Previous studies have determined that the number of platelets and platelet microparticles, derived from activated platelets, were abundant in the RA synovial fluid $(17,18)$. Additionally, as a concentrated source of autologous platelets, PRP contains various growth factors and cytokines, including platelet-derived growth factor, transforming growth factor- $\beta$ and insulin-like growth factor- 1 . These have been used for the treatment of bone defects, tendinopathies and intra-articular pathology of the synovial joints (19-21). Given the importance of the migration and invasion of RA-FLSs for the pathogenesis of RA, the effect of PRP on these processes has not been fully elucidated. Therefore, the aim of the present study was to investigate the effect of PRP on the migration and invasion of human RA-FLSs. It was determined that PRP may promote RA-FLS cell-matrix adhesion, migration and invasion. In addition, PRP may alter cell motility by cytoskeleton rearrangement and upregulation of the expression levels of matrix metalloproteinase-1 (MMP-1).

\section{Materials and methods}

Cell culture. RA-FLSs, obtained from GuangZhou Jennio Biotech Co., Ltd. (Guangzhou, China) were cultured in Dulbecco's modified Eagle's medium (DMEM; Gibco; Thermo Fisher Scientific, Inc., Waltham, MA, USA), supplemented with $10 \%$ fetal bovine serum (FBS; Invitrogen; Thermo Fisher Scientific, Inc.) and antibiotics $(100 \mathrm{mg} / \mathrm{ml}$ streptomycin and $100 \mathrm{U} / \mathrm{ml}$ penicillin) in a humidified incubator at $37^{\circ} \mathrm{C}$ with $5 \% \mathrm{CO}_{2}$. Cells used for experiments were at passage 3-6.

PRP preparation. PRP was harvested from $10 \mathrm{ml}$ cell culture (platelet concentration, $1.2 \times 10^{12} / 1$; Red Cross Blood Station of Yangzhou City, Yangzhou, China). The PRP samples were incubated with $10 \%$ calcium chloride and $100 \mathrm{U} / \mathrm{ml}$ bovine thrombin (Sigma-Aldrich, St. Louis, MO, USA), aggregated overnight at $4^{\circ} \mathrm{C}$, then centrifuged at $5,000 \mathrm{x}$ g for $20 \mathrm{~min}$ at $4^{\circ} \mathrm{C}$. The supernatant was collected and stored at $-20^{\circ} \mathrm{C}(16)$.

Scratch assay. Two different concentrations of PRP (2 and 5\%) were selected for the present study. RA-FLS were serum-starved for $24 \mathrm{~h}$, then plated in a 24-well plate. The cells were then incubated with culture medium in the absence (normal control; NC) or presence of PRP (2 and 5\%) for $48 \mathrm{~h}$. The cell monolayer was scraped in a straight line to create a 'scratch' using a P200 pipet tip. Subsequently, the cells were washed twice with phosphate-buffered saline (PBS). Photographs of the treated cells migrating within the scratch were captured at 0 and $24 \mathrm{~h}$. Each experiment was performed three times.

Transwell cell migration and invasion assays. Cell migration in vitro was performed using $6.5 \mathrm{~mm}$ Transwell chambers with $8 \mu \mathrm{m}$ pores (Corning, Corning, NY, USA). RA-FLSs seeded at a density of $2.0 \times 10^{4}$ were serum-starved for $24 \mathrm{~h}$, then seeded in the upper chamber of DMEM containing 2\% FBS. The lower chamber contained DMEM and 10\% FBS with or without PRP ( 2 or 5\%). The cells were incubated for $24 \mathrm{~h}$ at $37^{\circ} \mathrm{C}$ in a humidified atmosphere, and the non-migrating cells were wiped with dry cotton swabs. Cells that migrated through the filter were fixed with methanol for 15 min and stained with $0.1 \%$ crystal violet for $20 \mathrm{~min}$. For the cell invasion assay, RA-FLSs were seeded at a density of $4.0 \times 10^{4}$ into Transwell inserts pre-coated with Matrigel (BD Biosciences, Franklin Lakes, NJ, USA; $100 \mu \mathrm{g} / \mathrm{ml}$ diluted with serum-free DMEM), and DMEM with or without PRP (2 or 5\%) was added to the lower chamber as mentioned above. Cell invasion was allowed to occur for $24 \mathrm{~h}$, and cells on the top membrane surface were removed with cotton swabs. The migrating or invading cells were counted from five randomly selected microscopic fields at a magnification of $\mathrm{x} 200$ per insert. Three independent experiments were performed.

Cell-matrix adhesion assay. Cell-matrix adhesion assay was performed as previously described with some modifications (13). Cells were seeded at a density of $1.0 \times 10^{5}$ cells/well in 24-well plates, which were coated with $100 \mu \mathrm{g} / \mathrm{ml}$ type I collagen (BD Biosciences) at $4^{\circ} \mathrm{C}$ overnight and blocked with $1 \%$ bovine serum albumin (BSA; Invitrogen; Thermo Fisher Scientific, Inc.) at $37^{\circ} \mathrm{C}$ for $1 \mathrm{~h}$. RA-FLSs were pretreated with or without PRP (2 or $5 \%$ ) for $48 \mathrm{~h}$, and suspended in the adhesion buffer containing DMEM supplemented with $0.1 \%$ BSA. The cells were then plated onto the collagen I-coated plates. They were then incubated for $20 \mathrm{~min}$ at $37^{\circ} \mathrm{C}$, and unattached cells were washed out three times with PBS. The number of the attached cells was counted from five randomly selected microscopic fields, at a magnification of $x 200$. The independent experiments were performed three times.

Immunofluorescence analysis. RA-FLSs were plated onto coverslips in 24-well plates and incubated for $48 \mathrm{~h}$ at $37^{\circ} \mathrm{C}$ with or without PRP (2 or 5\%) in DMEM supplemented with $10 \%$ FBS. Next, the cells were washed with PBS and fixed with $4 \%$ paraformaldehyde diluted in PBS for $30 \mathrm{~min}$. The cells were permeabilized with $0.2 \%$ Triton X-100 in PBS for 10 min and blocked with $3 \%$ BSA in PBS for $1 \mathrm{~h}$. The cells were incubated with the rhodamine-conjugated phalloidin probe (Sigma-Aldrich) for $1 \mathrm{~h}$ at $37^{\circ} \mathrm{C}$ in the dark, and then washed three times with PBS. All cells were counterstained with 4',6-diamidino-2-phenylindole (DAPI; Sigma-Aldrich) for the nucleus. Following three additional washes with PBS, the cells were examined under a fluorescence microscope, and images were captured using Optronics digital camera (Optronics, Goleta, CA, USA).

Reverse transcription-quantitative polymerase chain reaction $(R T-q P C R)$. Total RNA was extracted using TRIzol (Vazyme Biotech Co., Ltd., Nanjing, China) according to the manufacturer's protocol, and cDNA was synthesized from RNA using HiScript QRT SuperMix for qPCR (Vazyme Biotech Co., Ltd.). RT-qPCR was performed using the Applied Biosystems 7500 Real-Time PCR System (Thermo Fisher Scientific, Inc.) using the AceQ qPCR SYBR Green Master mix (Vazyme Biotech Co., Ltd.) according to the manufacturer's protocols. The thermocycling conditions were as follows: $95^{\circ} \mathrm{C}$ for $5 \mathrm{~min}$; and 40 cycles of $95^{\circ} \mathrm{C}$ for $10 \mathrm{sec}$ and $60^{\circ} \mathrm{C}$ for $34 \mathrm{sec}$. Glyceraldehyde-3-phosphate dehydrogenase (GAPDH) was used as the internal control. The sequences of primers used 
A
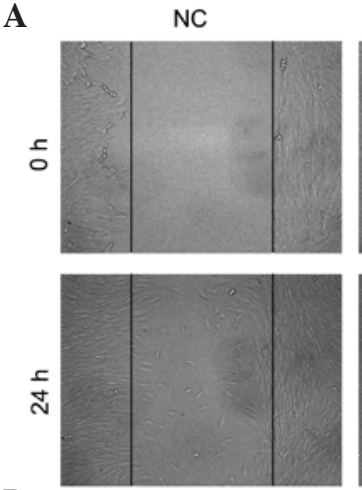

$\mathbf{B}$

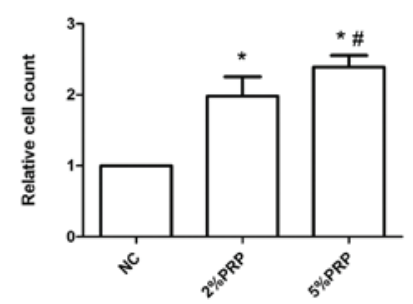

$5 \%$ PRP
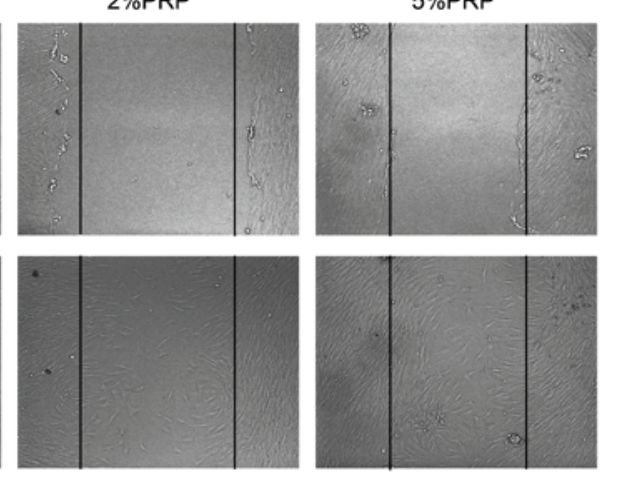

.

Fure 1.PRP promotes migration of RA-FLSs. The scratch assay determined that there is increased migration of RA-FLSs treated with PRP. (A) Images were captured at 0 and $24 \mathrm{~h}$ following the creation of the scratch. Magnification, $\mathrm{x} 40$. (B) Migratory cells treated with PRP were significantly increased compared with that of the NC group and in the $5 \%$ group compared with the $2 \%$ group. ${ }^{*} \mathrm{P}<0.05$ vs. the NC group and ${ }^{\#} \mathrm{P}<0.05$ vs. the $2 \% \mathrm{PRP}$ group. Data are presented as the mean \pm standard deviation, $\mathrm{n}=3$. NC, normal control; PRP, platelet-rich plasma; RA-FLSs, rheumatoid arthritis fibroblast-like synoviocytes.

were as follows: MMP-1, forward (F) 5'-ACTCTGGAGTAA TGTCACACCT-3' and reverse (R) 5'-GTTGGTCCACCTTT CATCTTCA-3'; GAPDH, F 5'-GCACCGTCAAGGCTGAGA AC-3' and R 5'-TGGTGAAGACGCCAGTGGA-3'. The relative levels of the MMP-1 mRNA normalized against GAPDH mRNA were evaluated using $2^{-\Delta \Delta C q}(22)$. All reactions were performed three times.

Western blot analysis. RA-FLSs were lysed in radioimmunoprecipitation assay (RIPA) buffer containing $50 \mathrm{mM}$ Tris/HCl ( $\mathrm{pH} 7.4), 150 \mathrm{mM} \mathrm{NaCl}, 1 \% \mathrm{NP}-40$, $0.5 \%$ sodium deoxycholate, $0.1 \%$ sodium dodecyl sulfate (SDS), $1 \mu \mathrm{mol}$ phenylmethylsulfonyl fluoride and a cocktail of protease inhibitors (Roche Diagnostics, Basel, Switzerland). The protein concentration was determined using a bicinchoninic acid assay. Subsequently, the proteins were resolved on $10 \%$ SDS-polyacrylamide gel electrophoresis (SDS-PAGE) and electro-transferred onto polyvinylidene fluoride membrane membranes (Roche Diagnostics). The membranes were subsequently blocked with PBS-Tween 20 containing $5 \%$ non-fat dried milk. Immunoblotting was performed using primary antibodies against MMP-1 (1:500; R\&D Systems, Inc., Minneapolis, MN, USA; cat. no. MAB901) and GAPDH (1:1,000; KangChen Bio-tech Co., Ltd., Shanghai, China; cat. no. KC-5G4) overnight at $4^{\circ} \mathrm{C}$, and the horseradish peroxidase-conjugated goat anti-mouse IG secondary antibody (1:2,000; Santa Cruz Biotechnology, Dallas, TX, USA; cat. no. sc-2301) incubated for $2 \mathrm{~h}$ at room temperature. Protein bands were detected by enhanced chemiluminescence (ECL) using the Pierce ECL Plus Western Blotting Substrate kit (Thermo Fisher Scientific, Inc.).

Statistical analysis. Data are presented as the mean \pm standard deviation. Statistical comparison between groups was performed using one-way analysis of variance followed by Dunnett's test using SPSS 19.0 software (IBM SPSS, Armonk, $\mathrm{NY}$, USA). $\mathrm{P}<0.05$ was considered to indicate a statistically significant difference.

\section{Results}

PRP promotes migration and invasion of RA-FLSs. RA-FLS migration and invasion are important to the pathophysiology of RA $(10,11)$. In order to determine the importance of PRP in RA-FLS migration, scratch and Transwell migration assays were performed. As presented in Fig. 1, the scratch assay highlighted a rapid movement of RA-FLSs. The migration front was evident at $24 \mathrm{~h}$, where a highly confluent monolayer region gradually migrated into the cell-free 'scratch' region, particularly in the 2 and 5\% PRP groups (Fig. 1A). The scratch assay determined that RA-FLSs incubated with 2 or $5 \%$ PRP have a significantly greater migration ability compared with the NC group ( $\mathrm{P}<0.05$; Fig. 1B). The Transwell migration assay revealed that the number of RA-FLSs across the polycarbonate membrane in the PRP group was significantly higher compared with the NC group ( $\mathrm{P}<0.05$; Fig. 2$)$. The number of migrating cells in the $5 \%$ PRP group was greater compared with the $2 \%$ PRP group (Fig. 2B), which was consistent with the results obtained with the scratch assay (Fig. 1). The aforementioned findings suggest that PRP enhanced the migration ability of RA-FLSs. Additionally, to evaluate the effect of PRP on the invasion of RA-FLSs, a Transwell invasion assay was performed. RA-FLSs were seeded into Matrigel precoated Transwell inserts, and allowed to invade for $24 \mathrm{~h}$. As presented in Fig. 2, the number of invasive cells in the chamber with PRP treatments was significantly higher compared with the NC group $(\mathrm{P}<0.05$; Fig. $2 \mathrm{C})$. The promotion of cell migration did not appear to be due to increased 
A
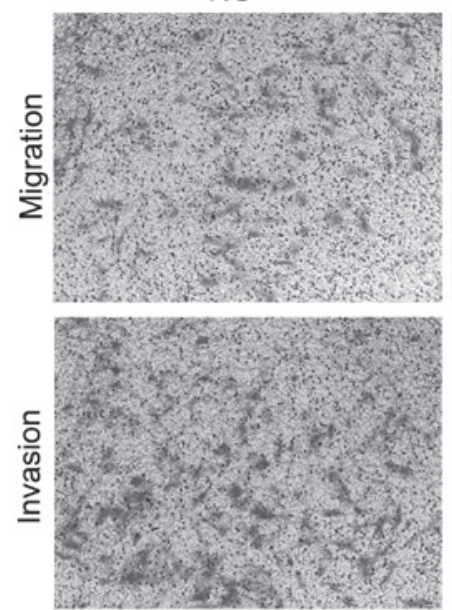

B

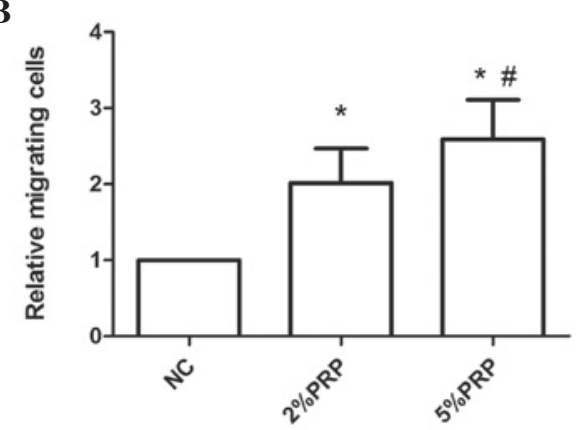

$2 \%$ PRP
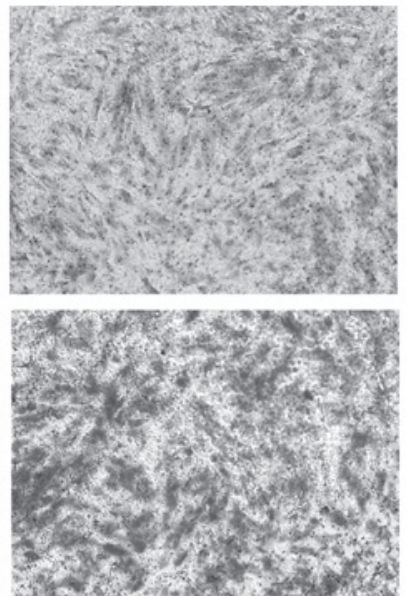

C

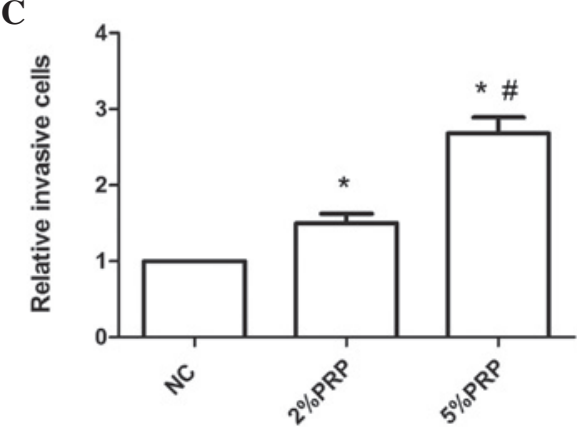

Figure 2. PRP promotes the migration and invasion of RA-FLSs. (A) Migratory and invasive RA-FLS on Transwell inserts were stained. Magnification, $\mathrm{x} 40$. (B) The number of migratory cells across the polycarbonate membrane in the PRP group was higher compared with the NC group and in the 5\% compared with the $2 \%$ group. (C) Invasion of RA-FLSs was promoted by PRP. A cell invasion assay was performed in Matrigel invasion chambers in 24 -well culture plates. Cells were incubated for $24 \mathrm{~h}$ and the Transwell inserts were stained with $0.1 \%$ crystal violet. The number of invasive cells was significantly increased compared with the $\mathrm{NC}$ group and in the $5 \%$ group compared with the $2 \%$ group. ${ }^{*} \mathrm{P}<0.05$ vs. the $\mathrm{NC}$ group and ${ }^{\#} \mathrm{P}<0.05$ vs. $2 \% \mathrm{PRP}$ group. All data are presented as the mean \pm standard deviation. NC, normal control; PRP, platelet-rich plasma; RA-FLSs, rheumatoid arthritis fibroblast-like synoviocytes.

A

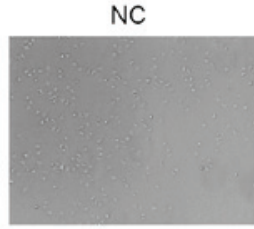

B

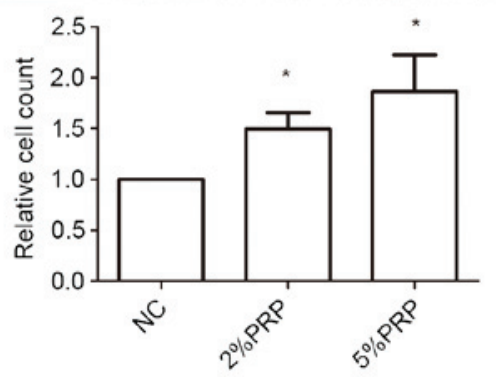

Figure 3. Effect of PRP on the adhesion of RA-FLS. (A and B) The number of attached cells was counted from five random microscopic fields per insert (magnification, $\mathrm{x} 200$ ). A total of three independent experiments was performed. Data are presented as the mean \pm standard deviation, $\mathrm{n}=3$. ${ }^{*} \mathrm{P}<0.05$ vs. NC group. NC, normal control; PRP, platelet-rich plasma; RA-FLSs, rheumatoid arthritis fibroblast-like synoviocytes.

cell viability (data not shown). Considered together, these findings demonstrated that PRP promoted migration and invasion of human RA-FLS.
PRP promotes RA-FLS adhesion onto the ECM. Cell adhesion and spreading to the ECM is a key step in the migration and invasion process of RA-FLSs. As the cells adhere and spread, they generate traction and thereby migrate on the substrate. To investigate the importance of PRP to the cell-matrix adhesion of RA-FLSs, they were seeded onto collagen I matrix for $20 \mathrm{~min}$. The cell adhesion ability on collagen I was significantly higher in PRP-treated cells compared with the NC group ( $\mathrm{P}<0.05$; Fig. 3 ). The number of adherent cells in $5 \%$ PRP group was higher compared with that in 2\% PRP group (Fig. 3). Therefore, it may be surmised that PRP enhanced cell-matrix adhesion.

PRP induces the reorganization of the actin cytoskeleton and formation of stress fibers and lamellipodia. The actin cytoskeleton with its polymerization dynamics is crucial for cell adhesion and migration (18). The present study investigated whether PRP may interfere with the actin cytoskeleton rearrangement and the production of filopodia and lamellipodia in RA-FLSs. Therefore, rhodamine-conjugated phalloidin was used to stain the filamentous actin (F-actin). PRP induced a significant decrease the number of centrally located stress fibers, and led to an increase in the formation of filopodia and lamellipodia in the detectable leading edge protrusions of RA-FLSs, particularly in the 5\% PRP group (Fig. 4). These 

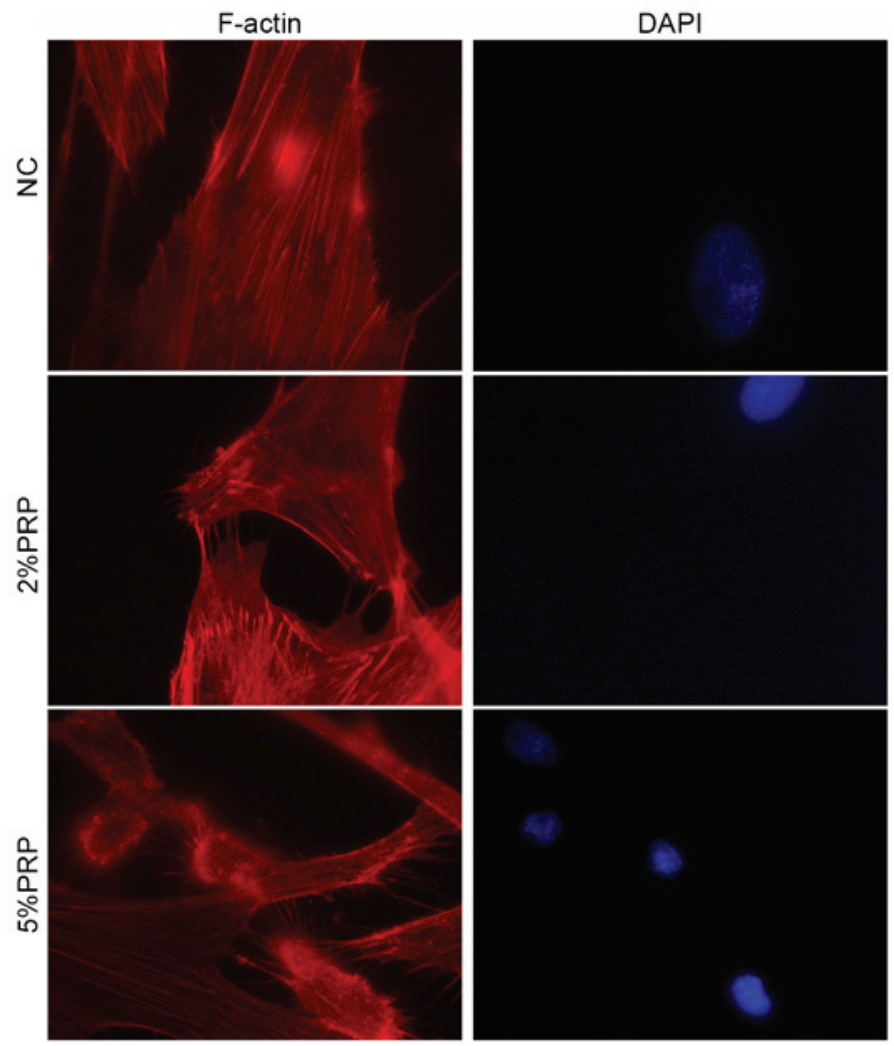

Figure 4. RA-FLSs were plated on glass coverslips and cultured in complete media for $48 \mathrm{~h}$. The cells were fixed, permeabilized and incubated with rhodamin-conjugated phalloidin. The images were captured with fluorescence microscopy. PRP induced a significant decrease in the number of centrally located stress fibers and led to an increase in the formation of filopodia and lamellipodia in the detectable leading edge protrusions in RA-FLSs. The images shown are representative of three individual experiments. Magnification, x1,000. NC, normal control; PRP, platelet-rich plasma; RA-FLSs, rheumatoid arthritis fibroblast-like synoviocytes; F-actin, filamentous actin.

A

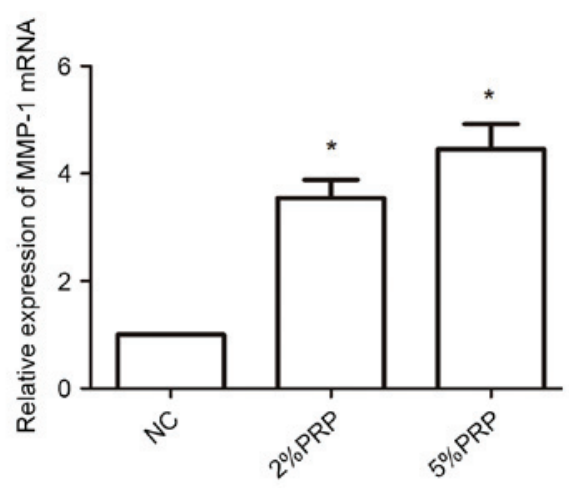

B

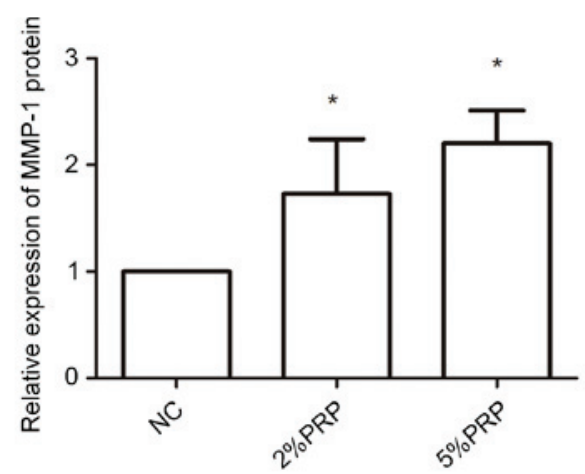

C

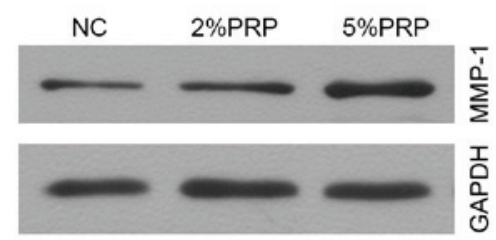

Figure 5. PRP promotes RA-FLS migration and invasion by increased expression levels of MMP-1. Expression levels of MMP-1 were determined by reverse transcription-quantitative polymerase chain reaction and western blot analysis. GAPDH was used as the internal control. MMP-1 (A) mRNA and $\left(\mathrm{B}\right.$ and C) protein expression levels were greater when PRP treatment was applied. Data are presented as the mean \pm standard deviation, $\mathrm{n}=3$. ${ }^{*} \mathrm{P}<0.05 \mathrm{vs}$. NC group. NC, normal control; PRP, platelet-rich plasma; MMP-1, matrix metalloproteinase-1; RA-FLS, rheumatoid arthritis fibroblast-like synoviocytes.

observations revealed that PRP is important for the dynamic reorganization of the actin cytoskeleton during adhesion and migration of RA-FLSs.
PRP upregulates the $M R N A$ and protein expression levels of MMP-1. MMP-1 is a proteolytic enzyme of collagen I, which is important for collagen remodeling during wound 
healing (23). PRP evidently promoted the migration and invasion of RA-FLSs; therefore, the underlying molecular mechanism was examined through a determination of the expression levels of MMP-1 (Fig. 5). The mRNA expression level of MMP-1 was significantly upregulated 3.546 \pm 0.34 -fold in RA-FLSs following treatment with $2 \%$ PRP compared with the NC group $(\mathrm{P}<0.05$; Fig. 5A), and 4.45 \pm 0.46 -fold in the 5\% PRP treatment group compared with $\mathrm{NC}(\mathrm{P}<0.05$; Fig. 5A). MMP-1 protein expression levels were also significantly greater in the PRP-treated groups compared with the $\mathrm{NC}$ group $(\mathrm{P}<0.05$; Fig. $5 \mathrm{~B}$ and $\mathrm{C})$. These findings indicate that MMP-1 may contribute to the migration and invasion of RA-FLSs when induced by PRP. The upregulation of mRNA and protein MMP-1 expression levels in RA-FLS exposed to PRP suggests that the enrichment of PRP in the synovial joints may be associated with RA, and the increased level of MMP-1 may be associated with catabolism of cartilage.

\section{Discussion}

The biological effects of PRP on various types of cell (chondrocytes, endothelial cells, osteoblasts, periodontal ligament, and so forth) have previously been demonstrated $(21,24)$. A previous study has determined that the number of platelets is associated with rheumatoid activity (16). Additionally, the platelets in PRP, subsequent to incubation with $\mathrm{CaCl}_{2}$ and thrombin, were demonstrated to secrete various growth factors and exhibit biological activities, including promoting regeneration, angiogenesis and wound healing $(25,26)$. To the best of our knowledge, the effects of PRP on RA-FLSs have not been previously reported. The present study demonstrated that PRP may promote migration and invasion of RA-FLSs. This conclusion is supported by the observation that PRP also promoted cell adhesion onto the ECM, interfered with the actin cytoskeleton rearrangement and upregulated the mRNA and protein expression levels of MMP-1.

RA usually arises in a few joints, and may spread to all joints during the course of the disease. RA-FLSs undergo the complex process of moving away from an affected joint, migrating into a healthy joint and subsequently invading the articular cartilage. During the pathogenesis of RA, FLSs may adhere onto the ECM, invade into the cartilage and bone, and activate osteoclasts to increase bone erosion and destruction, which are regarded as important mechanisms that lead to the invasion of cartilage and bone $(27,28)$. In order to investigate whether PRP affects RA-FLS migration and invasion, scratch, Transwell migration and invasion assays were performed. It was determined that PRP promoted RA-FLS migration, invasion and adhesion.

Cell adhesion and migration are complex and interdependent cellular processes. The movement of cells requires adhesion onto the ECM. As PRP is important for the positive regulation of the migration and invasion of RA-FLSs, it was determined that PRP may promote this migration through the regulation of the cell-matrix adhesion. A cell-matrix adhesion assay was performed, and it was revealed that PRP significantly facilitated the adhesion of RA-FLSs to the cell matrix. The present study determined that PRP may be a positive regulator in all three processes of cell-matrix adhesion, cell migration and cell invasion for RA-FLSs. The promotion of migration and invasion of RA-FLSs was triggered by PRP, possibly due to the increase of their adhesion to the cell-matrix.

The reorganization of cytoskeleton proteins, including F-actin, has been associated with cell migration and invasion. Migration of cells through the ECM is a multistep process, which commences with the extension of lamellipodia. The formation of actin stress fibers is frequently associated with cell adhesion, whereas membrane ruffling and filopodia and lamellipodia formation are associated with cell migration (29). Exposure of RA-FLSs to PRP resulted in marked changes, characterized by alterations in the cell shape and actin cytoskeleton. In order to confirm the effect of PRP on the migration and invasion of RA-FLSs, F-actin reorganization was assessed following treatment with PRP. It was determined that PRP resulted in a decreased assembly of actin stress fibers and an increased filopodia and lamellipodia formation in RA-FLSs. The current study suggested that the facilitation of cell adhesion and motility by PRP may be due to the regulation of actin reorganization and the formation of filopodia and lamellipodia.

MMP-1 is a proteolytic enzyme of collagen I that is important for collagen remodeling during wound healing (23). High expression levels of MMP-1 have been associated with the increased invasive ability of several types of cancer, including pancreatic, gastric, colorectal and breast cancer $(30,31)$. MMP-1 is vital for tissue remodeling, tumor progression and metastasis due to its proteolytic activities that aid in ECM degradation, invasion and cytokine mobilization $(32,33)$. The current study determined that PRP upregulated the mRNA and protein expression levels of MMP-1. Therefore, it is possible that PRP promoted the migration and invasion of RA-FLSs due to its capacity to upregulate MMP-1 expression levels. The upregulation of MMP-1 in RA-FLSs that have been exposed to PRP suggests that the application of PRP to synovial joints may be associated with increased catabolism of cartilage and extracellular matrix proteins.

In conclusion, the present study determined that PRP promoted the migration and invasion of RA-FLSs, and it may therefore be a novel target to limit the destruction of joints that is associated with patients with RA. The regulation of cell migration, invasion and adhesion on the ECM may be modulated through the induction of actin cytoskeleton reorganization and increased MMP-1 expression levels. Further studies are required to clarify the complicated molecular mechanism of PRP-induced cell migration and invasion in FLSs, and to elucidate the signaling mechanism behind this. Migration of RA-FLSs to the cartilage and bone has been considered to be the critical step in the aggravation of RA, and regulating the migration and invasion of RA-FLSs may be a novel therapeutic strategy to limit the destructive progress of RA.

\section{Acknowledgements}

The present study was supported by the National Nature Science Foundation of China (grant no. 81470070).

\section{References}

1. Karouzakis E, Neidhart M, Gay RE and Gay S: Molecular and cellular basis of rheumatoid joint destruction. Immunol Lett 106: 8-13, 2006. 
2. Müller-Ladner U, Pap T, Gay RE, Neidhart M and Gay S: Mechanisms of disease: The molecular and cellular basis of joint destruction in rheumatoid arthritis. Nat Clin Pract Rheumatol 1: 102-110, 2005.

3. Bartok B and Firestein GS: Fibroblast-like synoviocytes: Key effector cells in rheumatoid arthritis. Immunol Rev 233: 233-255, 2010.

4. Gravallese EM: Bone destruction in arthritis. Ann Rheum Dis 61 (Suppl 2): ii84-ii86, 2002.

5. Looney RJ: B cell-targeted therapy for rheumatoid arthritis: An update on the evidence. Drugs 66: 625-639, 2006.

6. Ma Y and Pope RM: The role of macrophages in rheumatoid arthritis. Curr Pharm Des 11: 569-580, 2005.

7. Skapenko A, Leipe J, Lipsky PE and Schulze-Koops H: The role of the T cell in autoimmune inflammation. Arthritis Res Ther 7 (Suppl 2): S4-S14, 2005.

8. Yasuda T: Cartilage destruction by matrix degradation products Mod Rheumatol 16: 197-205, 2006.

9. Klareskog L, Catrina AI and Paget S: Rheumatoid arthritis. Lancet 373: 659-672, 2009.

10. Huber LC, Distler O, Tarner I, Gay RE, Gay S and Pap T: Synovial fibroblasts: Key players in rheumatoid arthritis. Rheumatology (Oxford) 45: 669-675, 2006.

11. Lefèvre S, Knedla A, Tennie C, Kampmann A, Wunrau C, Dinser R, Korb A, Schnäker EM, Tarner IH, Robbins PD, et al: Synovial fibroblasts spread rheumatoid arthritis to unaffected joints. Nat Med 15: 1414-1420, 2009.

12. Tolboom TC, van der Helm-Van Mil AH, Nelissen RG, Breedveld FC, Toes RE and Huizinga TW: Invasiveness of fibroblast-like synoviocytes is an individual patient characteristic associated with the rate of joint destruction in patients with rheumatoid arthritis. Arthritis Rheum 52: 1999-2002, 2005.

13. Karouzakis E, Gay RE, Gay S and Neidhart M: Epigenetic control in rheumatoid arthritis synovial fibroblasts. Nat Rev Rheumatol 5: 266-272, 2009.

14. Tolboom TC, Pieterman E, van der Laan WH, Toes RE, Huidekoper AL, Nelissen RG, Breedveld FC and Huizinga TW: Invasive properties of fibroblast-like synoviocytes: Correlation with growth characteristics and expression of MMP-1, MMP-3, and MMP-10. Ann Rheum Dis 61: 975-980, 2002.

15. Marx RE: Platelet-rich plasma (PRP): What is PRP and what is not PRP? Implant Dent 10: 225-228, 2001.

16. Wang F, Wang NS, Yan CG, Li JH and Tang LQ: The significance of platelet activation in rheumatoid arthritis. Clin Rheumatol 26: 768-771, 2007.

17. Endresen GK: Evidence for activation of platelets in the synovial fluid from patients with rheumatoid arthritis. Rheumatol Int 9: 19-24, 1989

18. Boilard E, Nigrovic PA, Larabee K, Watts GF, Coblyn JS, Weinblatt ME, Massarotti EM, Remold-O'Donnell E, Farndale RW, Ware J and Lee DM: Platelets amplify inflammation in arthritis via collagen dependent microparticle production. Science 327: 580-583, 2010 .
19. Han J, Meng HX, Tang JM, Li SL, Tang Y and Chen ZB: The effect of different platelet-rich plasma concentration on proliferation and differentiation of human periodontal ligament cells in vitro. Cell Prolif 40: 241-252, 2007.

20. Anitua E, Andia I, Ardanza B, Nurden P and Nurden AT: Autologous platelets as a source of proteins for healing and tissue regeneration. Thromb Haemost 91: 4-15, 2004.

21. Kanno T, Takahashi T, Tsujisawa T, Ariyoshi W and Nishihara T: Platelet-rich plasma enhances human osteoblast-like cell proliferation and differentiation. J Oral Maxillofac Surg 63: 362-369, 2005.

22. Livak KJ and Schmittgen TD: Analysis of relative gene expression data using real-time quantitative PCR and the 2(-Delta Delta C(T)) method. Methods 25: 402-408, 2001

23. Shin MK, Lee JW, Kim YI, Kim YO, Seok H and Kim NI: The effects of platelet-rich clot releasate on the expression of MMP-1 and type I collagen in human adult dermal fibroblasts: PRP is a stronger MMP-1 stimulator. Mol Biol Rep 41: 3-8, 2014.

24. Kakudo N, Morimoto N, Kushida S, Ogawa T and Kusumoto K: Platelet-rich releasate promotes angiogenesis in vitro and in vivo. Med Mol Morphol 47: 83-89, 2014.

25. Kakudo N, Minakata T, Mitsui T, Kushida S, Notodihardjo FZ and Kusumoto K: Proliferation-promoting effect of platelet-rich plasma on human adipose-derived stem cells and human dermal fibroblasts. Plast Reconstr Surg 122: 1352-1360, 2008

26. Kakudo N, Kushida S, Minakata T, Suzuki K and Kusumoto K: Platelet-rich plasma promotes epithelialization and angiogenesis in a split thickness skin graft donor site. Med Mol Morphol 44: 233-236, 2011.

27. Volin MV, Huynh N, Klosowska K, Chong KK and Woods JM: Fractalkine is a novel chemoattractant for rheumatoid arthritis fibroblast-like synoviocyte signaling through MAP kinases and Akt. Arthritis Rheum 56: 2512-2522, 2007.

28. Gravallese EM, Manning C, Tsay A, Naito A, Pan C, Amento E and Goldring SR: Synovial tissue in rheumatoid arthritis is a source of osteoclast differentiation factor. Arthritis Rheum 43: $250-258,2000$

29. Akakura S and Gelman IH: Pivotal role of AKAP12 in the regulation of cellular adhesion dynamics: Control of cytoskeletal architecture, cell migration, and mitogenic signaling. J Signal Transduct 2012: 529179, 2012.

30. Murray GI, Duncan ME, O'Neil P, Melvin WT and Fothergill JE: Matrix metalloproteinase-1 is associated with poor prognosis in colorectal cancer. Nat Med 2: 461-462, 1996.

31. McGowan PM and Duffy MJ: Matrix metalloproteinase expression and outcome in patients with breast cancer: Analysis of a published database. Ann Oncol 19: 1566-1572, 2008.

32. Lu X, Wang Q, Hu G, Van Poznak C, Fleisher M, Reiss M, Massagué J and Kang Y: ADAMTS1 and MMP1 proteolytically engage EGF-like ligands in an osteolytic signaling cascade for bone metastasis. Genes Dev 23: 1882-1894, 2009.

33. Pei D: Matrix metalloproteinases target protease-activated receptors on the tumor cell surface. Cancer Cell 7: 207-208, 2005. 